\title{
Cardioprotection of Rosuvastatin Pre-conditioning on Myocardial Ischemia / Reperfusion Injury in a Rat Model
}

\author{
Pei-Xi Zhang ${ }^{1}$
}

https://orcid.org/0000-0002-4708-3566

Hong-Xia Zhang ${ }^{2}$

https://orcid.org/0000-0001-7000-9113

Dai-Liang $\mathrm{Hou}^{2}$

https://orcid.org/0000-0002-4298-754X

\section{Zhi-Chao Xiao ${ }^{1}$}

https://orcid.org/0000-0003-2539-8564

\author{
Cheng-Yun Zhou ${ }^{1, *}$ \\ https://orcid.org/0000-0002-6478-0945
}

1Jining Medical University, Jining No 1 People's Hospital, Department of Cardiac Surgery, Jining City, Shandong, People's Republic of China; ${ }^{2}$ Jining Medical University, Jining No 1 People's Hospital, Department of Anesthesiology, Jining City, Shandong, People's Republic of China.

Received: 2019.02.01; Accepted: 2019.07.05.

* Correspondence: doctorzcy@163.com, Department of Cardiac Surgery, Jining No 1 People's Hospital of Jining Medical University, Jining City, Shandong, 272000, People's Republic of China.

\section{HIGHLIGHTS}

- Rosuvastatin pre-conditioning protects the heart against I/R injury.

- Rosuvastatin pre-conditioning attenuates oxidative stress and cell apoptosis in the I/R heart.

\footnotetext{
Abstract: This study aimed to investigate the cardioprotection of rosuvastatin pre-conditioning (R-Pre) in a rat model of myocardial ischemia / reperfusion (I/R). Male SD rats were assigned into three groups: sham group, I/R group and R-Pre group. Rats in I/R group and R-Pre group received ischemia for $30 \mathrm{~min}$ and reperfusion for $2 \mathrm{~h}$. In R-Pre group, rats received intragastrical administration with rosuvastatin at $5 \mathrm{mg} / \mathrm{kg}$ once daily for 1 week. After 2-h reperfusion, the cardiac function was detected by ultrasonography; the blood was collected for biochemical analysis; the heart was collected for the TUNEL staining and immunohistochemistry for Bcl-2 and Bax. Our results showed rosuvastatin
} 
pre-conditioning for 1 week could significantly reduce the infarct ratio and improve the cardiac function after myocardial I/R injury, in which attenuation of oxidative stress and cell apoptosis played an important role. Our study provides evidence on the cardioprotection of rosuvastatin pre-conditioning and highlight the use of rosuvastatin before cardiopulmonary bypass.

Keywords: Myocardial ischemia/reperfusion; rosuvastatin; oxidative stress; apoptosis; pre-conditioning

\section{INTRODUCTION}

The introduction of cardiopulmonary bypass (CPB) has allowed cardiac surgery to develop. Since it was introduced in 1953, CPB techniques have significantly improved and most patients can tolerate the procedure relatively well. However, the heart is isolated from the circulation during the cardiac surgery with CPB and thus susceptible to suffer from an ischemic insult followed by an additional hit upon reperfusion, which is also known as ischemia / reperfusion (I/R) injury [1]. To date, some measures have been taken to prevent the myocardial injury during $\mathrm{CPB}$, including preparation of the myocardium for $\mathrm{CPB}$, modulation of inflammatory response, regulation of myocardial oxygen consumption and myocardial conditioning. In recent years, ischemic conditioning (pre- and post-) [2,3], remote ischemic pre-conditioning [4] and pharmacological conditioning [5] have been found to be cardioprotective on heart I/R injury.

Statins are a group of 3-hydroxy-3-methylglutaryl coenzyme A (HMG CoA) reductase inhibitors and can competitively inhibit HMG CoA reductase and induce the expression of low density lipoprotein (LDL) receptors within the liver, thereby increasing the catabolism of plasma LDL and consequently decreasing LDL-cholesterol [6]. Some large clinical trials have revealed the cardioprotection of statins, which is ascribed not only their cholesterol lowering effect, but also to the cholesterol-lowering independent effects [7]. These effects include preserving endothelial function, stabilizing arterial plaque, scavenging free radicals, and inhibiting proliferation, inflammation, and apoptosis [8]. A meta-analysis of 13 randomized studies showed statin pre-treatment was associated with reduced peri-procedural myocardial infarction and a $44 \%$ risk reduction in major adverse cardiovascular events at 30-d irrespective of clinical presentation [9], and similar findings were also found in recent studies [10,11]. The American College of Cardiology, American Heart Association, and European Society of Cardiology have suggested that statins should be used as the secondary prevention following acute myocardial infarction (AMI). Rosuvastatin is a fully synthetic statin. Although a variety of studies have showed the cardioprotection of rosuvastatin, a recent study showed perioperative statin therapy failed to prevent postoperative atrial fibrillation or perioperative myocardial damage in patients undergoing elective cardiac surgery and acute kidney injury was more common in rosuvastatin treated patients [12].

In this study, myocardial ischemia / reperfusion model was established in rats, and the cardioprotection of rosuvastatin was investigated, and the potential mechanisms were explored.

\section{MATERIAL AND METHODS}

\section{Animals}

A total of 36 healthy male Sprague Dawley rats weighing 250-350 g were purchased from the Experimental Animal Center of Jining Medical Collage. Animals were housed in a specific pathogen free environment at $22-25^{\circ} \mathrm{C}$ with $12 \mathrm{~h} / 12 \mathrm{~h}$ light-dark cycle and given ad libitum access to food and water. This study was approved by the First People's Hospital of 
Jining City and all the procedures were performed according to the Guideline for the Animal Care and Use in China.

\section{Grouping and treatments}

Animals were randomly divided into three groups: sham group $(n=10)$, l/R group $(n=13)$ and rosuvastatin pre-conditioning (R-Pre) group $(n=13)$. In the sham group, animals received intragastric treatment with normal saline once daily for 1 week and then were subjected to thoracotomy without myocardial ischemia; in I/R group, animals received intragastric treatment with normal saline once daily for 1 week and then were subjected to 30-min myocardial ischemia and 2-h reperfusion after thoracotomy; in R-Pre group, animals received intragastric treatment with rosuvastatin at $5 \mathrm{mg} / \mathrm{kg}$ and then were subjected to 30-min myocardial ischemia and 2-h reperfusion after thoracotomy.

Myocardial I/R injury was performed according to previously reported ${ }^{13}$. In brief, rats were anesthetized by intraperitoneal administration of ketamine $(100 \mathrm{mg} / \mathrm{kg})$ and xylazine $(10 \mathrm{mg} / \mathrm{kg})$. After endotracheal intubation, controlled ventilation was performed with a rodent ventilator (Suzhou Experimental Instrument Co., Ltd, China) with room air, and the body temperature was maintained within a physiologic range using a heating pad. A left thoracotomy was performed and the pericardium was opened. Then, a 6-0 nonabsorbable nylon suture was passed around the left descending coronary artery. The ends of the suture were threaded through a propylene tube to form a snare which was clamped to induce myocardial ischemia. Successful ligation was confirmed by regional cyanosis and contraction loss. After 30-min ischemia, reperfusion was allowed by releasing the snare. Pleural space was evacuated and the chest was closed in 3 layers. The rats were allowed to recover from anesthesia and given ad libitum access to water and food. The survival rate was assessed within 2-h reperfusion.

\section{Cardiac function assessment}

After 2-h reperfusion, the survived animals were subjected to cardiac function assessment by ultrasonography with a high-resolution ultrasound system for small animal imaging (Vevo 2100; VisualSonics Inc., Toronto, ON, Canada). The left ventricular end diastolic diameter (LVIDd), left ventricular end systolic diameter (LVIDs), ejection fraction (EF) and left ventricular fraction shortening (LVFS) were determined.

\section{Triphenyltetrazolium chloride staining}

After 2-h reperfusion, the hearts were subsequently harvested, frozen, sliced, and incubated with $1 \% 2,3,5$-triphenyltetrazolium chloride (TTC) at $37^{\circ} \mathrm{C}$ for $20 \mathrm{~min}$ in dark. After being fixed in $10 \%$ paraformaldehyde, the infarct area was measured using the Image-Pro Plus 6.0 software (Media Cybernetics, Silver Spring, MD, USA). Each section was scanned using an HP Scanjet 4890 (HP, Palo Alto, CA, USA). The infarct size was measured using ImageJ software (National Institutes of Health, USA) and reported as a percent of the total left ventricular area..

\section{Biochemical examinations}

After 2-h reperfusion, the survived animals were sacrificed after intraperitoneal anesthesia with sodium pentobarbital at $60 \mathrm{mg} / \mathrm{kg}$. Then, laparotomy was performed, and blood was collected from the abdominal aorta. Blood was centrifuged at $3000 \mathrm{rpm}$ for $5 \mathrm{~min}$, and the serum was harvested and stored at $-80^{\circ} \mathrm{C}$ for further detection of GSH-Px, SOD, MDA, NO, CKMB and cTnl.

Glutathione peroxidase (GSH-PX) activity was measured with GSH-PX assay kit (Nanjing Jiancheng Biotech Co, Ltd.,) according to the manufacturer's instructions and expressed as units per milliliter $(\mathrm{U} / \mathrm{ml})$. The absorbance was measured at $412 \mathrm{~nm}$ in a 
microplate reader. Total myocardial superoxide dismutase (SOD) activity was determined using Xanthine Oxidase Assay Kits (Nanjing Jiancheng Biotech Co, Ltd.,) according to the manufacturer's instructions and expressed as units per milliliter $(\mathrm{U} / \mathrm{ml})$. The absorbance was measured at $550 \mathrm{~nm}$ in a microplate reader.

The myocardial malondialdehyde (MDA) and nitric oxide (NO) contents were measured with MDA assay kit and NO assay kit (Nanjing Jiancheng Biotech Co, Ltd.,), respectively, according to the manufacturer's instructions and expressed as nmol per milliliter $(\mathrm{nmol} / \mathrm{ml})$.

The myocardial enzymes (CKMB and cTnl) were measured with kits from Jieluoxuan Biotech Co, Ltd; \#SEA478Ra) according to the manufacturer's instructions and expressed as $\mathrm{ng}$ per milliliter $(\mathrm{ng} / \mathrm{ml})$ and $\mathrm{pg}$ per milliliter $(\mathrm{pg} / \mathrm{ml})$, respectively.

\section{TUNEL staining}

After 2 -h reperfusion, the survived animals were sacrificed as above mentioned. The heart was harvested, fixed in $4 \%$ paraformaldehyde, embedded in paraffin and cut into $4-\mu \mathrm{m}$ sections. For quantification of cell apoptosis, terminal deoxynucleotidyl transferase dUTP nick end labeling (TUNEL) staining was performed using in situ Apoptosis Detection Kit (Roche, USA) according to the manufacturer's instructions. The number of TUNEL-positive neurons was manually counted in six randomly selected sections from each animal at a magnification of $200 x$, and an average was calculated. Data were presented as the ratio of TUNEL-positive cells (\%).

\section{Immunohistochemistry for Bcl-2 and Bax}

Myocardial sections were obtained as above mentioned. Then, these sections were deparaffinized with xylene and treated with an ethanol gradient $(100 \%, 95 \%, 85 \%$, and $75 \%$ ethanol). After antigen retrieval, sections were washed in PBS thrice (5 min for each) and then treated with $\mathrm{H}_{2} \mathrm{O}_{2}$, followed by washing in PBS thrice ( 5 min for each). After blocking in $5 \%$ bovine serum albumin (BSA) for $30 \mathrm{~min}$ at room temperature, sections were incubated with primary antibody (Bcl-2 [ab196495, Abcam, UK; 1:100] and Bax [ab32503, Abcam, UK; $1: 150]$ ) at $4^{\circ} \mathrm{C}$ over night. After washing in PBS thrice (5 min for each), sections were incubated with HRP conjugated secondary antibody (Abcam, UK). Visualization was done with $\mathrm{DAB}$, followed by counterstaining with haematoxylin. After transparentization, sections were mounted, and observed under a light microscope. The Bcl-2 or Bax positive cells were counted.

\section{Statistical analysis}

Statisitcal analysis was performed with SPSS version 12.0 (SPSS, LA, USA). One way analysis of variance (ANOVA) was employed for the comparisons among groups, followed by Student-Newman-Keuls method. Data are expressed as mean \pm standard deviation (SD). $A$ value of $P<0.05$ was considered statistically significant.

\section{RESULTS}

\section{Survival rate}

Within 2-h reperfusion, 3 rats in I/R group and 2 rats in R-Pre group died, but none died in sham group. There was no significant difference in the survival rate among three groups.

\section{Myocardial infarction}

After 2-h reperfusion, animals were sacrificed and the heart was collected for the detection of infarct ratio after TTC staining. Results showed the infarct ratio was $0 \%$ in sham group, $31.6 \%$ in I/R group and $12.3 \%$ in R-Pre group, and significant difference in the infarct ratio was observed among three groups. Paired comparisons also showed R-Pre significantly reduced the infarct ration ( $P<0.05$ vs $\mathrm{I} / \mathrm{R}$ group) (Figure 1$)$. 

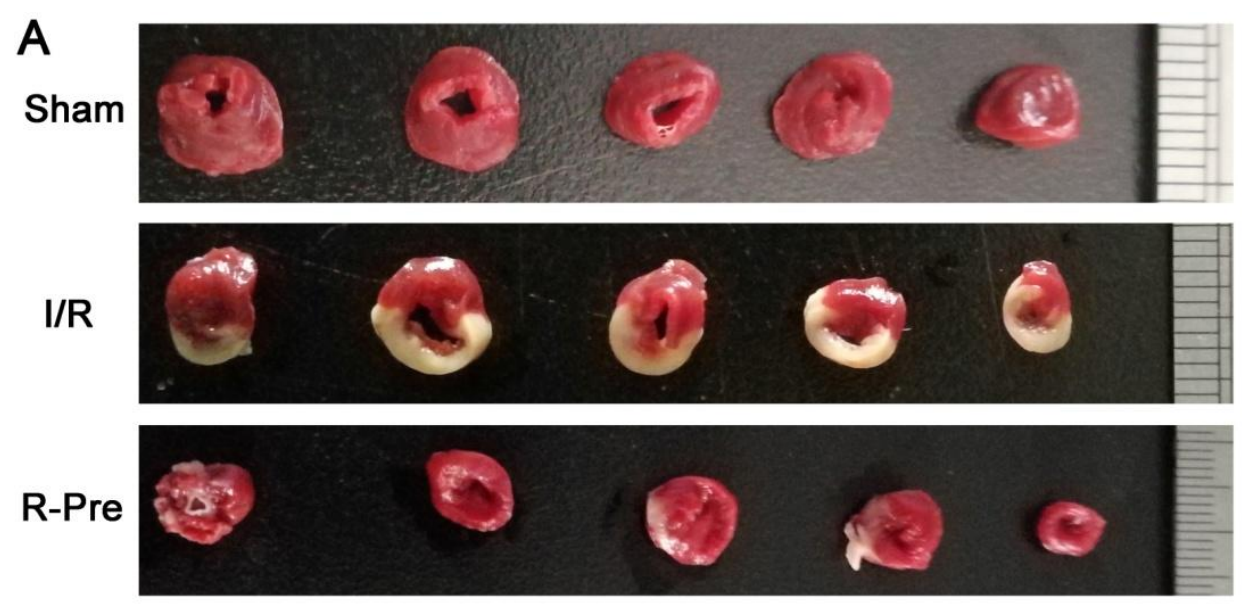

B

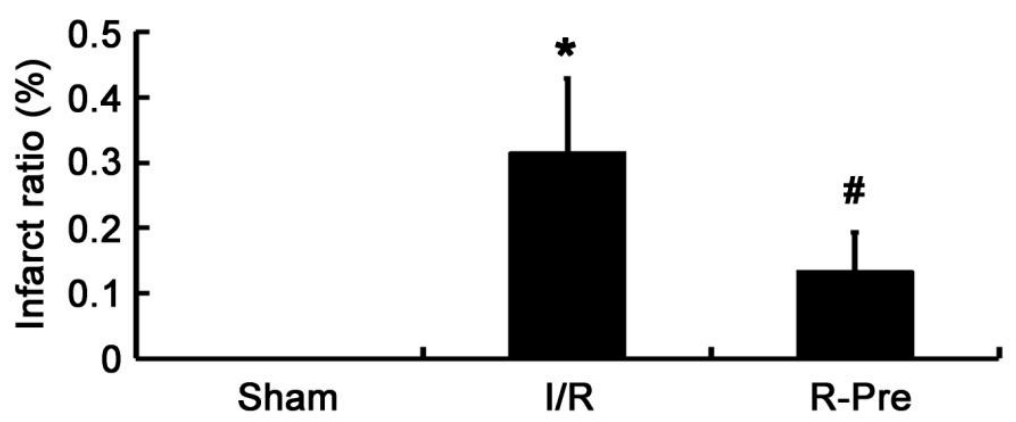

Figure 1 Infarct ratio of the myocardium in different groups.

A. TTC staining of the heart after I/R injury; B. Infarct ratio in different group ${ }^{*} \mathrm{P}<0.05$ vs sham group; ${ }^{\mathrm{P}} \mathrm{P}<0.05$ vs $\mathrm{l} / \mathrm{R}$ group

\section{Biochemical parameters}

Blood was collected at predesigned time points, and the contents of GSH-Px, SOD, MDA, NO, CKMB and cTnl were detected. Results showed I/R significantly increased the MDA content, but markedly reduced GSH-Px, SOD and NO contents, which were accompanied by the pronounced increases in CKMB and cTnl ( $\mathrm{P}<0.05$ vs sham group). In the R-Pre group, the MDA content was markedly lower than in I/R group, and the GSH-Px, SOD and NO contents increased significantly as compared to I/R group $(P<0.05)$ (Figure 2$)$. 

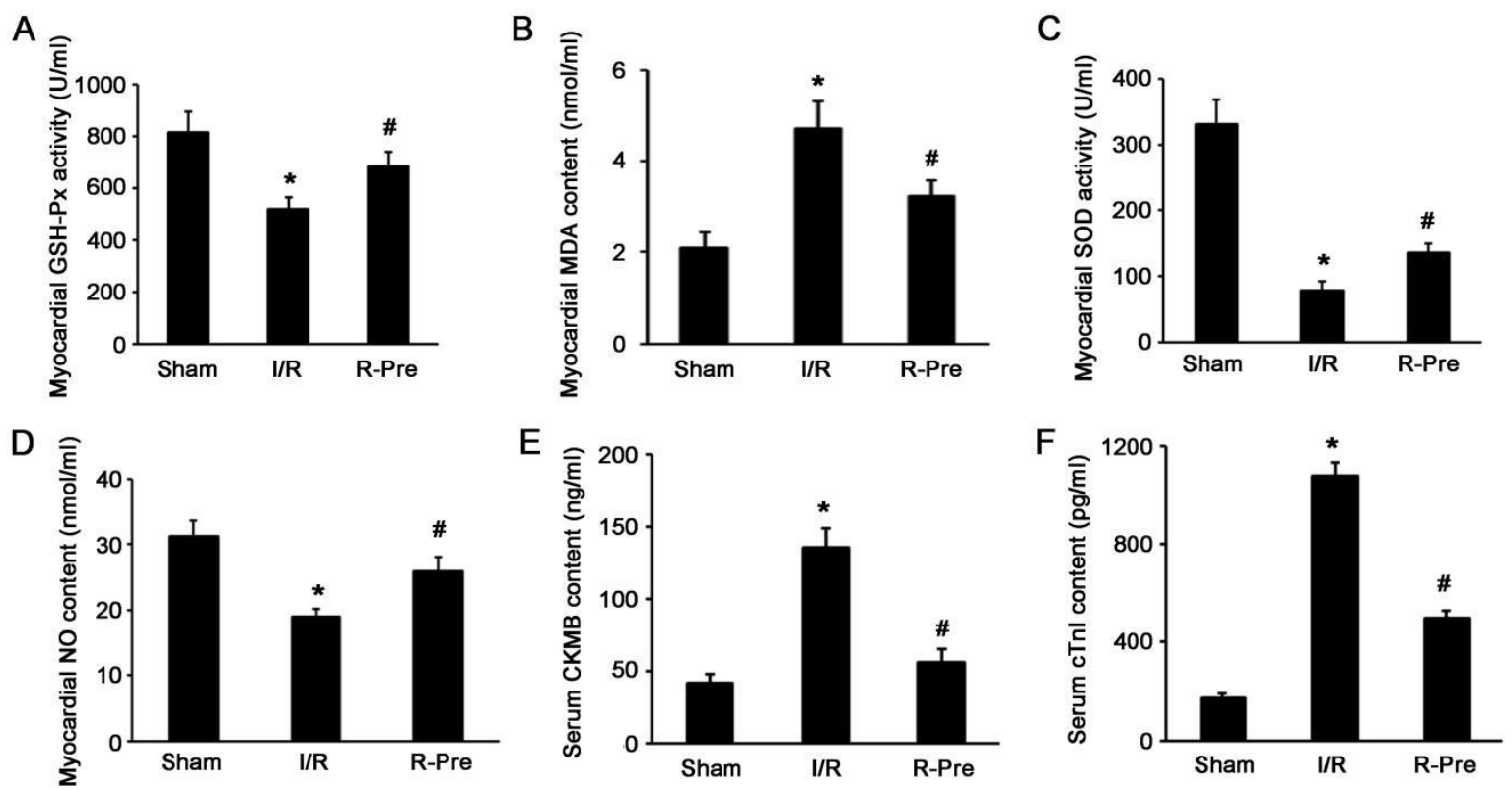

Figure 2 Oxidative stress and myocardial enzymes in different groups

A. GSH-Px activity; B. MDA content; C. SOD activity; D. NO content; E. CKMB content; F. cTnl content. ${ }^{*} \mathrm{P}<0.05$ vs sham group; \#P<0.05 vs $\mathrm{I} / \mathrm{R}$ group

\section{TUNEL staining}

TUNEL staining was performed to assess myocyte apoptosis following I/R. Only $5.6 \pm 1.5 \%$ of myocytes were apoptotic in sham group, but the apoptotic cells accounted for $50.8 \pm 8.3 \%$ after $\mathrm{I} / \mathrm{R}$ group ( $\mathrm{P}<0.05$ vs sham group). In the $\mathrm{R}$-Pre group, the proportion of apoptotic cells was markedly lower than in I/R group $(22.5 \pm 4.8 \% ; P<0.05)$ (Figure 3 ). 


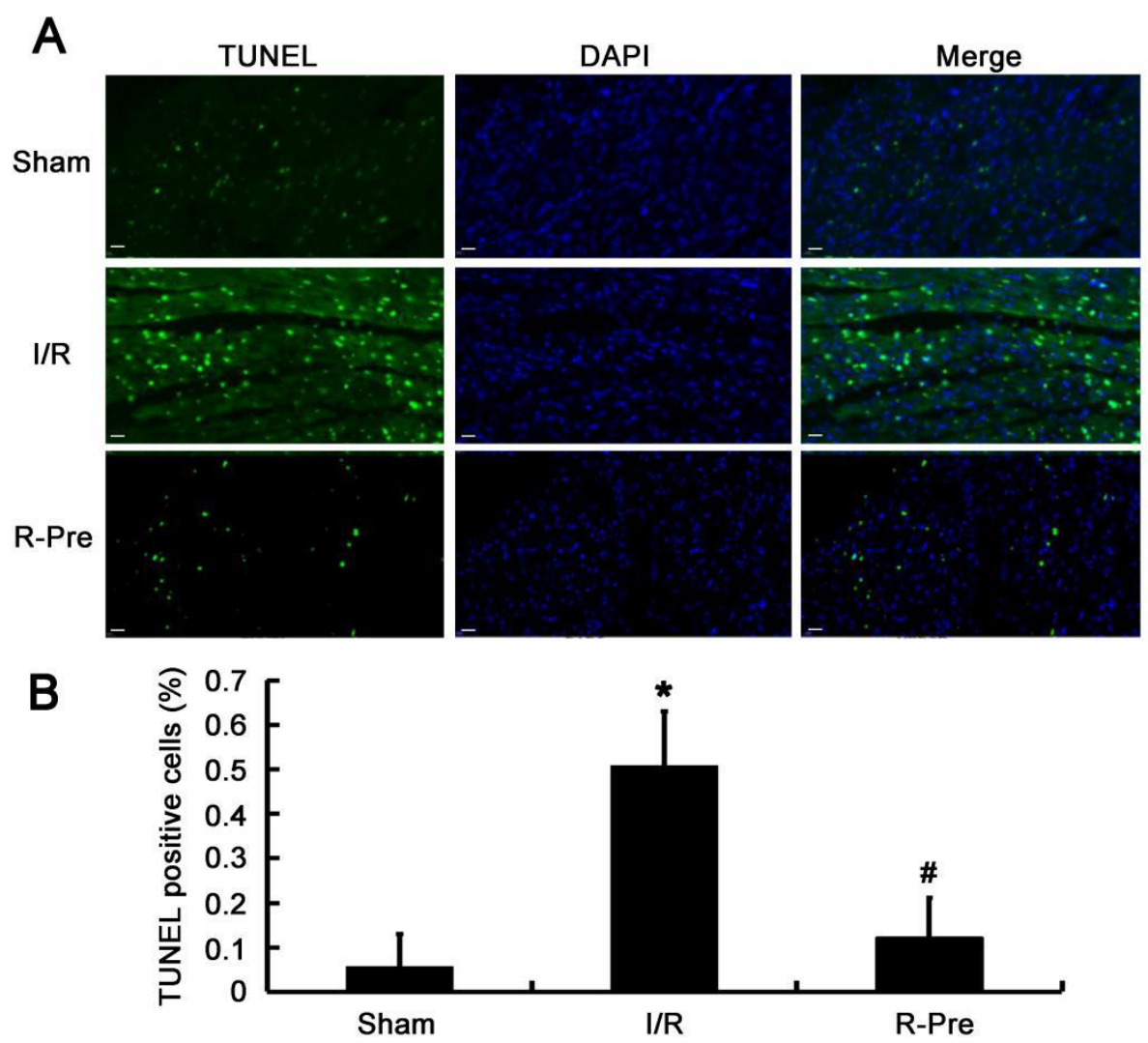

Figure 3 Apoptotic cells in the myocardium of different groups

A. TUNEL staining of the myocardium in different groups; B. quantification of apoptotic cells in the myocardium of different groups. ${ }^{*} \mathrm{P}<0.05$ vs sham group; ${ }^{*} \mathrm{P}<0.05$ vs I/R group

\section{Immunohistochemistry}

Immunohistochemistry was done to detect the expression of $\mathrm{Bcl}-2$ and $\mathrm{Bax}$, two proteins in the apoptosis pathway, in the myocardium. The integrated optical density (IOD) of Bax was $0.23 \pm 0.08$ in sham group, $0.95 \pm 0.28$ in $\mathrm{l} / \mathrm{R}$ group and $0.62 \pm 0.17$ in R-Pre group, and significant difference was noted in the IOD of Bax among three groups. Paired comparisons showed the IOD in R-Pre group increased significantly as compared to $\mathrm{I} / \mathrm{R}$ group $(\mathrm{P}<0.05)$. The IOD of $\mathrm{Bcl}-2$ was $0.84 \pm 0.22$ in sham group, $0.21 \pm 0.06$ in $\mathrm{I} / \mathrm{R}$ group and $0.58 \pm 0.18$ in R-Pre group, and significant difference was noted in the IOD of Bcl-2 among three groups. Paired comparisons also showed marked difference in the IOD of $\mathrm{Bcl}-2$ between I/R group and R-Pre group $(\mathrm{P}<0.05)$ (Figure 4). 


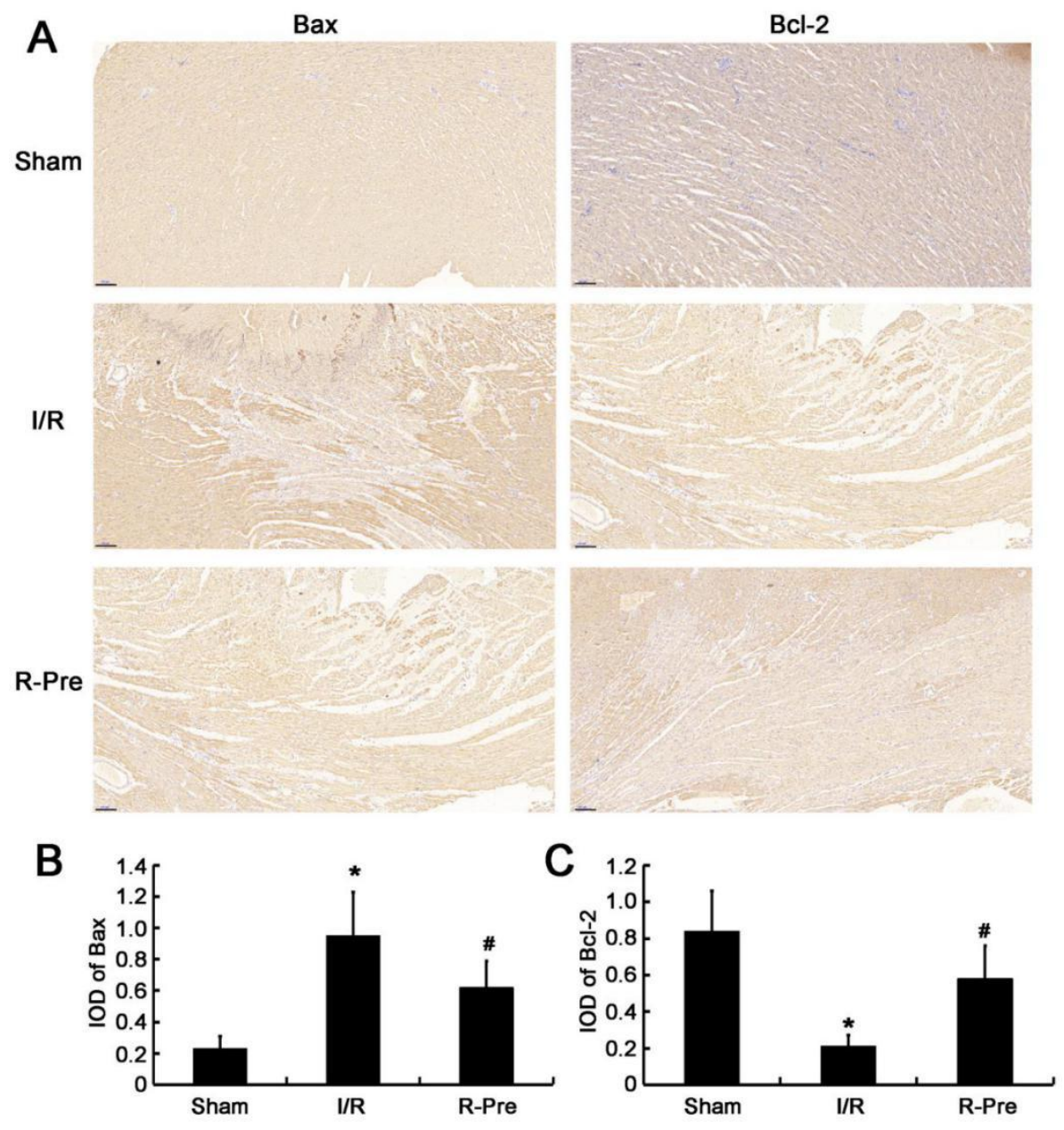

Figure 4 Expression of Bcl-2 and Bax expression in the myocardium of different groups

A. Immunohistochemistry for Bcl-2 and Bax in the myocardium; B. Quantification of $\mathrm{Bcl}-2$ and Bax expression (integrated optical density [IOD]) in the myocardium. ${ }^{*} \mathrm{P}<0.05$ vs sham group; ${ }^{*} \mathrm{P}<0.05$ vs I/R group

\section{Cardiac function}

Myocardial function was measured after 2-h reperfusion. Results showed the LVIDd increased significantly but the LVID, EF, and FS reduced markedly after I/R. In Pre-R group, the LVIDd remained unchanged as compared to I/R group, but the LVID, EF and EF increased significantly in Pre-R group were markedly higher than those in $I / R$ group $(P<0.05)$ (Figure 5). 

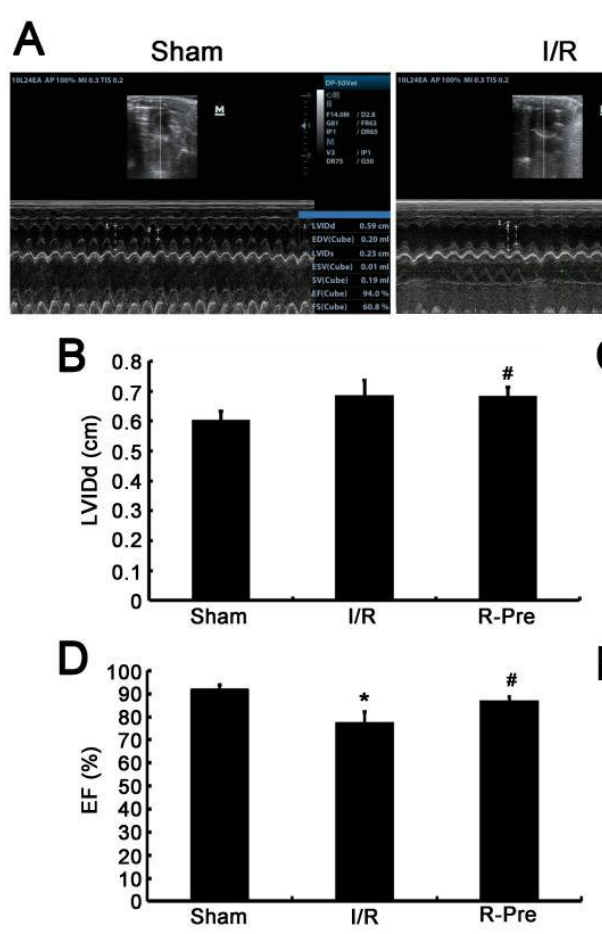

I/R

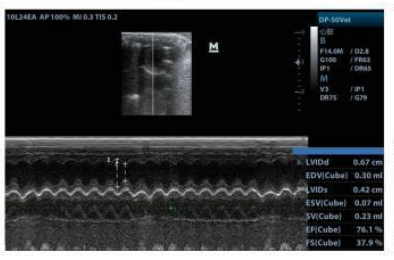

C
R-Pre

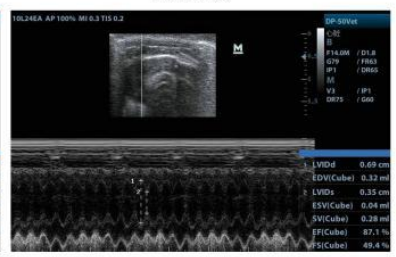

E

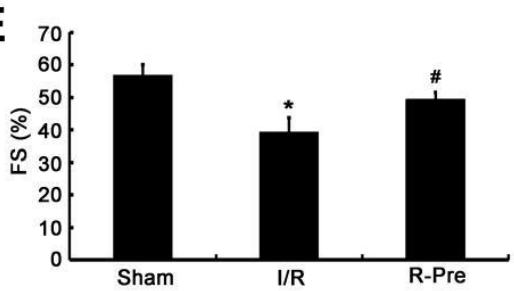

Figure 5 Cardiac function in different groups

Ultrasonography of the heart after I/R injury in different groups; B. LVIDd in different groups; C. LVID in different groups; D. EF in different groups; E. FS in different groups. ${ }^{*} \mathrm{P}<0.05$ vs sham group; ${ }^{\#} \mathrm{P}<0.05$ vs $\mathrm{I} / \mathrm{R}$ group

\section{DISCUSSION}

Rosuvastatin is a fully synthetic lipid-lowering agent and one of the two most commonly used statins with potent, effective and safe HMG-COA reductase inhibition [14]. The Food and Drug Administration has approved its use for adults patients with dyslipidemia, and rosuvastatin at a low dose was found to be more effective in reducing cardiovascular risk as compared to other statins (simvastatin, atorvastatin and pravastatin), proving to be the most powerful agent in CV risk reduction [15,16]. As in other statins, rosuvastatin also has pleiotropic effects. It ancillary properties are involved in reducing the oxidative stress, inflammation, improving endothelial function and inhibiting cell apoptosis [17-19].

In recent years, there is evidence showing another effect of rosuvastatin: prevention of periprocedural $\mathrm{MI}$ in patients undergoing to percutaneous coronary intervention $(\mathrm{PCI})$ and CPB. A single $40 \mathrm{mg}$ loading dose of rosuvastatin administrated before $\mathrm{PCl}$ in patients with acute coronary syndrome (ACS) or within $24 \mathrm{~h}$ prior elective $\mathrm{PCl}$ has shown to reduce the incidence of periprocedural myocardial necrosis [20,21]. In pigs, periprocedural statin reloading therapy was found to enhance myocardial and coronary function after cardiac surgery with CPB [22]. In patients, receiving CPB, $20 \mathrm{mg} /$ day of rosuvastatin was administered between preoperative day 7 and postoperative day 28, and results showed it could reduce complications in certain (especially intraoperative) cases of systemic inflammatory response caused by the CPB technique [23]; in addition, statin $(20 \mathrm{mg} / \mathrm{d})$ pretreatment for 1 week before surgery also reduced myocardial damage after coronary surgery and could improve both short- and long-term results [24]. However, these studies mainly focused on the inflammation response induced by CPB. In this study, myocardial I/R injury was induced by clamping the coronary artery to mimic the $\mathrm{I} / \mathrm{R}$ injury induced by CPB, and the oxidative stress and apoptosis were investigated. Our results also showed rosuvastatin pre-treatment for 1 week before myocardial I/R could significantly attenuate 
myocardial injury (reductions in infarct ratio and myocardial enzymes) and improve the cardiac function (LIVD, EF, and FS). As above mentioned, the ancillary properties of rosuvastatin are related to the anti-oxidation, anti-inflammation, anti-apoptosis, endothelial protection and other activities. Then, we further investigated the potential mechanisms. As shown in a majority of studies, myocardial I/R significantly increased the oxidative stress and the apoptotic cells, but in the presence of rosuvastatin pre-treatment, the oxidative stress was significantly attenuated, and the apoptotic cells reduced markedly as compared to rosuvastatin free animals. As compared to previous studies, rosuvastatin was administered once or twice. However, in the present study, received rosuvastatin pre-treatment for 1 week. In recent years, there is evidence showing that statins may increase the risk of developing type 2 diabetes mellitus (DM) as off-target effect, but the the risk is low both in absolute terms and when compared with the reduction in coronary events [25]. Moreover, 1-week rosuvastatin treatment is still insufficient to increase this risk due to the short-term treatment. Although rosuvastatin has other adverse effects as in other statins, very few cases are reported in literature [7].

However, there were still limitations in this study. First, only one dose of rosuvastatin was used in the present study, and it is better to compare the efficacy of rosuvastatin at different doses in preventing cardiac injury secondary to myocardial I/R. Second, the specific mechanism underlying the cardioprotection of rosuvastatin pre-treatment was not further investigated. In addition, there is evidence showing that rosuvastatin may improve the myocardial remodeling after ischemia [26]. In the present study, the long term consequence of cardiac function and the myocardial remodeling are still unclear, and more studies are needed to elucidate these issues.

\section{CONCLUSION}

Taken together, rosuvastatin pre-treatment for 1 week may reduce myocardial infarct ratio and improve the cardiac function, which may be ascribed to the attenuation of oxidative stress and myocyte apoptosis after myocardial I/R injury.

Funding: This research was funded by Medical and Health Technology Development Project of Shandong Province, grant number 2016WS0152".

Acknowledgments: We thank the staff in the Department of Pathology, Jining No 1 People's Hospital of Jining Medical University.

Conflicts of Interest: The authors declare no conflict of interest. The funders had no role in the design of the study; in the collection, analyses, or interpretation of data; in the writing of the manuscript, and in the decision to publish the results.

\section{REFERENCES}

1. De Hert $S$, Moerman A. Myocardial injury and protection related to cardiopulmonary bypass. Best Pract Res Clin Anaesthesiol. 2015;29:137-49.

2. Wu ZK, livainen T, Pehkonen E, Laurikka J, Tarkka MR. Ischemic preconditioning suppresses ventricular tachyarrhythmias after myocardial revascularization. Circulation. 2002;106:3091-6.

3. Durdu S, Sirlak M, Cetintas D, Inan MB, Eryılmaz S, Ozcinar E, et al. The efficacies of modified mechanical post conditioning on myocardial protection for patients undergoing coronary artery bypass grafting. J Cardiothorac Surg. 2012;7:73.

4. Brevoord D, Hollmann MW, De Hert SG, van Dongen EH, Heijnen BG, de Bruin A, et al. Effect of remote ischemic conditioning on atrial fibrillation and outcome after coronary artery bypass grafting (RICO-trial). BMC Anesthesiol 2011;11:11. 
5. Kaneko T, Saito Y, Hikawa Y, Yasuda K, Makita K. Dose-dependent prophylactic effect of nicorandil, an ATP-sensitive potassium channel opener, on intra-operative myocardial ischaemia in patients undergoing major abdominal surgery. Br J Anaesth. 2001;86:332-7.

6. Istvan ES. Structural mechanism for statin inhibition of 3-hydroxy-3-methylglutaryl coenzyme A reductase. Am Heart J. 2002;144:S27-32.

7. Cortese F, Gesualdo M, Cortese A, Carbonara S, Devito F, Zito A, et al. Rosuvastatin: Beyond the cholesterol-lowering effect. Pharmacol Res. 2016;107:1-18.

8. Rosenson RS. Pluripotential mechanisms of cardioprotection with HMG-CoA reductase inhibitor therapy. Am J Cardiovasc Drugs. 2001;1:411-20.

9. Patti G, Cannon CP, Murphy SA, Mega S, Pasceri V, Briguori $C$ et al. Clinical benefit of statin pretreatment in patients undergoing percutaneous coronary intervention: a collaborative patient-level meta-analysis of 13 randomized studies. Circulation. 2011;123:1622-32.

10. An J, Shi F, Liu S, Ma J, Ma Q. Preoperative statins as modifiers of cardiac and inflammatory outcomes following coronary artery bypass graft surgery: a meta-analysis. Interact Cardiovasc Thorac Surg. 2017;25:958-65.

11. Lampropoulos K, Megalou A, Bazoukis G, Tse G, Manolis A. Pre-loading therapy with statins in patients with angina and acute coronary syndromes undergoing $\mathrm{PCl}$. $\mathrm{J}$ Interv Cardiol. 2017;30:507-13.

12. Zheng Z, Jayaram R, Jiang L, Emberson J, Zhao Y, Li Q, et al. Perioperative Rosuvastatin in Cardiac Surgery. N Engl J Med. 2016;374:1744-53.

13. Lemoine S, Blanchart K, Souplis M, Lemaitre A, Legallois D, Coulbault L, et al. Argon Exposure Induces Postconditioning in Myocardial Ischemia-Reperfusion. J Cardiovasc Pharmacol Ther. 2017;22:564-73.

14. Wander GS, Hukkeri MYK, Yalagudri S, Mahajan B, Panda AT. Rosuvastatin: Role in Secondary Prevention of Cardiovascular Disease. J Assoc Physicians India. 2018;66(3):65-9.

15. Davidson $\mathrm{MH}$. Rosuvastatin safety: lessons from the FDA review and post-approval surveillance. Expert Opin Drug Saf. 2004;3:547-57.

16. Ballantyne CM, Bertolami M, Hernandez Garcia HR, Nul D, Stein EA, et al. Achieving LDL cholesterol, non-HDL cholesterol, and apolipoprotein B target levels in high-risk patients: Measuring Effective Reductions in Cholesterol Using Rosuvastatin therapY (MERCURY) II. Am Heart J. 2006;151:975.e1-9.

17. Cortese F, Gesualdo M, Cortese A, Carbonara S, Devito F, Zito A, et al. Rosuvastatin: Beyond the cholesterol-lowering effect. Pharmacol Res. 2016;107:1-18.

18. Park DS, Yun KH, Park HY, Rhee SJ, Kim NH, Oh SK, et al. Antioxidative Activity after Rosuvastatin Treatment in Patients with Stable Ischemic Heart Disease and Decreased High Density Lipoprotein Cholesterol. Korean Circ J. 2016;46:309-14.

19. Yen CH, Sun CK, Leu S, Wallace CG, Lin YC, Chang LT, et al. Continuing exposure to low-dose nonylphenol aggravates adenine-induced chronic renal dysfunction and role of rosuvastatin therapy. J Transl Med. 2012;10:147.

20. Yun KH, Jeong MH, Oh SK, Rhee SJ, Park EM, Lee EM, et al. The beneficial effect of high loading dose of rosuvastatin before percutaneous coronary intervention in patients with acute coronary syndrome. Int J Cardiol. 2009;137:246-51.

21. Cay S, Cagirci G, Sen N, Balbay Y, Durmaz T, Aydogdu S. Prevention of periprocedural myocardial injury using a single high loading dose of rosuvastatin. Cardiovasc Drugs Ther. 2010;24:41-7.

22. Kuhn EW, Liakopoulos OJ, Deppe AC, Slottosch I, Neef K, Sterner-Kock A, et al. Rosuvastatin reloading before cardiac surgery with cardiopulmonary bypass. Eur Surg Res. 2013;50:1-13.

23. Ozguler IM, Burma O, Uysal A, Akbulut H. Rosuvastatin Lowers Systemic Inflammatory Response in Coronary Artery Bypass Graft Accompanied by Cardiopulmonary Bypass Surgery: A Randomized Controlled Study. Clin Invest Med. 2015;38:E154-63. 
24. Mannacio VA, lorio D, De Amicis V, Di Lello F, Musumeci F. Effect of rosuvastatin pretreatment on myocardial damage after coronary surgery: a randomized trial. $\mathrm{J}$ Thorac Cardiovasc Surg. 2008;136:1541-8.

25. Sattar N, Preiss D, Murray HM, Welsh P, Buckley BM, de Craen AJ, et al. Statins and risk of incident diabetes: a collaborative meta-analysis of randomised statin trials. Lancet. 2010;375:735-42.

26. Wang W, Zhang Y, Gao M, Wang J, Hou Y. Effect of rosuvastatin on atrial structural remodeling in rabbits with myocardial infarction. Biomed Rep. 2015;3:78-82. (C) $(5)$
EY 2018 by the authors. Submitted for possible open access publication
under the terms and conditions of the Creative Commons Attribution (CC
BY NC) license (https://creativecommons.org/licenses/by-nc/4.0/). 